\title{
Conventional Tracheostomy Versus Percutaneous Tracheostomy: A Retrospective Study
}

\author{
Praveen Kumar Thakur ${ }^{1}$ Anil Kumar Jain ${ }^{1} \cdot$ Tahir Ali Khan $^{2} \cdot$ Sanyogita Jain $^{3}$
}

Received: 31 March 2018/Accepted: 6 April 2018/Published online: 10 April 2018

(C) Association of Otolaryngologists of India 2018

\begin{abstract}
Objectives Emergency Tracheostomy is a life saving surgical procedure. With the advent of newer instruments and equipments in the critical care units, there is marked improvement in the quality of care of the critically ill patients and such patients are able to survive for a longer period. Elective tracheostomy is being done in those patients who needs positive pressure ventilation, for a longer duration. Objectives of our study are to compare conventional tracheostomy (CT) to percutaneous tracheostomy (PcT) and to identify the strategy with the lowest frequency of potentially life threatening events. Study Design Retrospective comparative study. Patients Included 30 patients who met inclusion criteria. Study Settings Tertiary care centre (medical college). Results 15 patients underwent CT and 15 underwent PcT. Blood loss, mean operation time and complications were compared. Blood loss and operation time was lesser in CT compared to PcT. There were no reported complications in both the techniques. Conclusion In our study CT took lesser time with lesser blood loss and without any complications. But statistically, this difference was not significant. Thus person with refined skill in the technique is of utmost importance in deciding the choice of a technique.
\end{abstract}

Anil Kumar Jain

jaindranil@gmail.com

1 Department of ENT, Chirayu Medical College and Hospital, Bhopal, M.P 462030, India

2 Department of Anesthesiology, Chirayu Medical College and Hospital, Bhopal, India

3 Department of Microbiology, Chirayu Medical College and Hospital, Bhopal, India
Keywords Tracheostomy - Conventional tracheostomy · Percutaneous tracheostomy - Critical care .

Intensive care unit · ICU

\section{Background}

General perception is that percutaneous tracheostomy is better than conventional tracheostomy and has various advantages over the latter, which is not always correct.

\section{Introduction}

Tracheostomy refers to a surgical procedure to create an opening through the anterior wall of neck into the trachea (windpipe). A tracheostomy tube (TT) is placed through this opening, to provide an airway and to remove secretions from the lungs. After this procedure, trachea is communicating with exterior. It is performed in Operation Theatre and also bed side in intensive care units. According to timing of performance of tracheostomy they may be classified into emergency and elective (planned) tracheostomy.

As per duration up to which TT needs to be kept in situ, it may be classified into temporary and permanent tracheostomy and as per site of stoma on anterior wall of tracheal, it may be classified into high, mid or low tracheostomy (with reference to isthmus of thyroid). The two main approaches are, Conventional Tracheostomy (CT) and Percutaneous Tracheostomy(PcT). In 1909, Chevalier Jackson standardized the surgical tracheostomy. With the continued improvement in the equipments and instruments in Intensive care units (ICUs), like positive pressure ventilators, monitors and medications, critically ill patients can be kept alive for longer period. Long term airway 
assistance/ventilator support by laryngotracheal intubation my lead to complications like tracheal wall necrosis, traceo-oesophageal fistula etc., and to avoid such complications, elective tracheostomy is a better choice.

In last few decades, methods to access trachea and to establish safe airway have improved. Guidewire technique (Seldinger technique) was adopted for percutaneous tracheostomy. In 1955, Shelden et al. [1], introduced the first modern tracheostomy. In his technique, cutting trochar was used, which often led to complications like lacerations of adjacent structures. Many advantages of PcT cited include minimal incision, less dissection resulting in reduced tissue trauma and hence fewer wound complications like bleeding, infection etc. [2].

\section{Ciaglia Serial Dilatation Technique}

Ciaglia et al. [3], first described the technique of PDT (Percutaneous Dilatation Tracheostomy) using serial dilators over a guide wire. This technique has undergone three major changes since then, in terms of level of tracheal interspace cannulation, use of concurrent bronchoscopy and use of a single tapered dilator. The site of insertion has moved caudal from cricoid cartilage by one or two tracheal interspaces.

\section{Ciaglia Single Dilator Tracheostomy}

It is popularly known as Ciaglia Blue Rhino (Cook Critical Care, Bloomington, IN, USA). It was introduced in 1999, more than a decade after initial description of Ciaglia technique. It is a simpler kit than the original kit and entails use of a single-beveled curved hydrophilic dilator. Use of single dilator is associated with reduced tidal volume loss during the procedure as change in dilator is not required.

\section{Balloon Dilatational Tracheostomy (Ciaglia Blue Dolphin, Cook Medical)}

In this technique inflation of a modified angioplasty balloon over a guidewire is used to dilate the trachea. The balloon is inflated with saline to 11 atmospheric pressure for $15 \mathrm{~s}$ to make tracheal stoma. Then, the balloon is deflated and the tracheostomy tube insertion is done in a single step. This is an advantage over conventional single dilator PDT where the dilator needs to be removed before inserting the tracheostomy tube. There is a presumption that balloon dilation minimizes the pressure on the tracheal wall as compared to other techniques. Schachner et al. [4] and Griggs et al. [5] developed different single guidewire dilating tracheostomy forceps.

When comparisons between CT and PcT studies are drawn, the CT publications cited are usually those published in the 1960s and 1970s [6-10]. When the comparison between the two techniques is done with studies conducted during the same period, the advantages of PcT are less obvious. With the availability of commercial sets, PcT can be performed by every competent physician with less assistance and as bed side procedure too, thus decreasing overall cost to the patient.

\section{Materials and Methods}

In our retrospective study, we included 30 patients, who met inclusion criteria. Out of these 30 patients, 15 underwent conventional tracheostomy (CT) and 15 underwent Percutaneous tracheostomy (PcT), from Aug. 2015 to Aug. 2016. The patients/family members of unconscious patients gave their informed consent. The age, sex, diagnosis, previous intubations, reasons for tracheostomy and drugs used were recorded. The type of tracheostomy technique to be used was randomized using a computer-generated random numbers table. The procedure was done either in the operating room or at the bedside in the intensive care unit. All standard surgical steps of Conventional Tracheostomy (CT) were followed. Position given. Local parts painting, draping done. Local anaesthetic agent infiltrated. Horizontal incision kept in skin crease midway between cricoid cartilage and suprasternal notch. Soft tissue and strap muscles dissected in midline. Thyroid gland isthmus was located and retracted. Trachea identified and confirmed by its appearance, tracheal rings and aspiration of air in fluid filled syringe. Pre tracheal fascia incised and dissected. Tracheotomy done. Cuffed tracheostomy tube inserted under direct vision. Position of tracheostomy tube in trachea confirmed and secured using neck straps.

For PcT, we used modified Ciaglia's technique. Horizontal skin crease incision was kept $2 \mathrm{~cm}$ above the sternal notch, to have the same postoperative status as with CT. The incision was carried only through the skin and subcutaneous fascia, without direct visualization of the trachea, before the needle puncture. Also, the trachea was opened at the level of the second or third tracheal ring and not below the cricoid cartilage. Finally, the entire procedure was done under bronchoscopic guidence [11].

All tracheostomies included in the study were performed by one of the authors, having extensive experience in the procedure. A standardized questionnaire was used to evaluate intraoperative variables. The morphology of the neck (normal, large, short) and thyroid gland (normal or enlarged), the size of the cutaneous incision, the duration of the procedure, the difficulty of the procedure and intraoperative complications were recorded. The duration of surgery was timed starting with the skin incision and ending when the tracheostomy tube was inserted. The surgeon 
evaluated the difficulty in surgery and the intraoperative bleeding on a scale of $0-2$. Perioperative complications, such as death, cardiorespiratory arrest, aspiration, pneumothorax, pneumomediastinum, laryngeal lesion, posterior tracheal wall lesion, subcutaneous emphysema and difficult cannula insertion, were recorded. Postoperative evaluation was performed daily during the first week and then on the 14th day by the same intensive care unit nurse, who was blinded to the tracheostomy technique used. Local status and complications from tracheostomy was assessed through a standardized questionnaire: death, pneumothorax, pneumomediastinum, aspiration, subcutaneous emphysema, hemorrhage and wound infection was recorded. Long-term evaluation was done 3 months after decannulation by the same surgeon. Patients were questioned about subjective dyspnea, dysphonia, or unesthetic scar. The surgeon evaluated the scar, using criteria such as color, level, and mobility. A flexible bronchoscope was used to perform direct laryngoscopy and was passed, when possible, through the vocal cords to evaluate the trachea. Perioperative complications covered the actual procedure and the following $48 \mathrm{~h}$. Postoperative complications included the remaining follow-up, up to 3 months after decanulation. For each group, complications were classified as serious, intermediate, or minor [12].

\section{Inclusion Criteria}

Endotracheal intubated patients who required prolonged ventilatory support in intensive care unit, above 18 years of age, normal laryngeal frame work and cervical soft tissue, palpable cricoid cartilage, normal cervical spine and normal coagulation parameters.

\section{Exclusion Criteria}

Emergency procedure, patients less than 18 years of age, infected cases, cervical spine fracture.

\section{Results}

The parameters that were recorded and compared were, duration of procedure (skin incision to insertion of T'stomy tube), intra operative bleeding and complications like emphysema, paratracheal insertion and pneumothorax) (Tables 1, 2, 3).

\section{Discussion}

In the present era continued efforts are being made to provide best possible treatment to each and every patient, including critically ill patients. Also with advancement in the instruments, equipments, monitors and medication along with better facilities for training of medical and paramedical staff,the bar of standards of management of critically ill patients is rising day by day. There is marked improvement in the quality of care to the critically ill patients and they are able to survive for a longer period. Also it is established that although emergency tracheostomy is a life saving surgical procedure, elective tracheostomy has its own role in management of patients in intensive care units. Elective tracheostomy is being done in those patients who needs positive pressure ventilator support, for a longer duration.

In our study, mean operation time in CT was $16.4 \mathrm{~min}$ and in PcT was 23.93 min. Suggesting lesser time was taken in CT compared to PcT. This is contrary to previous studies in which mean operation time was more in CT compared to PcT. This may be due to better instruments in OT and strict adherence to trained and same operating surgeon. Griffen et al. have reported operating time of 4.3-21 $\mathrm{min}$ for percutaneous and 13.5-60 $\mathrm{min}$ for open tracheostomy. Donaldson et al. [13] have reported a mean operative time of 13.2 min for percutaneous tracheostomy and $24.2 \mathrm{~min}$ for conventional tracheostomy.

Also mean blood loss in patients who underwent Conventional Tracheostomy $(6.8 \mathrm{ml})$ was lesser compared to those who underwent Percutaneous Tracheostomy $(8.06 \mathrm{ml})$. This may be due to better illumination in operation theatre and availability of unipolar and bipolar electric cautry.

In our study, in conventional tracheostomy, 01 patient developed surgical emphysema and 01 patient developed pneumothorax. Both patients were managed successfully. In percutaneous tracheostomy group, in 01 patient there was paratracheal insertion and needed to convert PcT into conventional tracheostomy. 01 patient had pneumothorax and 01 patient developed surgical emphysema. Both these patient were managed conservatively and outcome was satisfactory. Follow up to 3 months was free of any further complications. Lesser complications may be due to better instrumentation and adequately trained staff in the operation theatre in Conventional Tracheostomy and availability of flexible video-bronchoscopes for direct vision and monitoring thus helping prevention of injury in the lumen of trachea and soft tissue.

Results of previous studies suggests that complications were less in PcT compared to Conventional Surgical Tracheostomy but complications when occured were serious 
Table 1 Operative time,Blood loss and complications during conventional tracheostomy

\begin{tabular}{llll}
\hline Patient no. & Operative time $(\min )$ & Blood loss (in ml) & Complications (Emphysema, pneumothorax, Paratracheal insertion) \\
\hline 1 & 11 & 06 & - \\
2 & 09 & - & - \\
3 & 27 & 08 & - \\
4 & $\mathbf{4 1}$ & $\mathbf{1 2}$ & Emphysema \\
5 & 12 & 05 & - \\
6 & 10 & 06 & - \\
7 & 08 & 08 & - \\
8 & 16 & 08 & - \\
9 & 22 & 08 & - \\
10 & 14 & 07 & - \\
11 & $\mathbf{0 4}$ & 04 & - \\
12 & 26 & 06 & Pneumothorax \\
13 & 31 & 10 & - \\
14 & 06 & 06 & - \\
15 & 09 & $\mathbf{0 4}$ & \\
\hline
\end{tabular}

Maximum and minimum time taken, are made bold

Table 2 Operative time,Blood loss and complications during percutaneous tracheostomy

\begin{tabular}{llll}
\hline Patient no. & Operative time $(\mathrm{min})$ & Blood loss (in ml) & Complications (Emphysema, pneumothorax, Paratracheal insertion) \\
\hline 16 & 28 & $\mathbf{0 4}$ & - \\
17 & 26 & 06 & - \\
18 & 32 & $\mathbf{1 6}$ & Pneumothorax \\
19 & 18 & 04 & - \\
20 & $\mathbf{5 4}$ & 12 & Paratracheal insertion \\
21 & 19 & 08 & - \\
22 & 24 & 14 & - \\
23 & 22 & 07 & - \\
24 & 26 & 06 & - \\
25 & 29 & 08 & - \\
26 & 24 & 04 & - \\
27 & 18 & 08 & - \\
28 & 16 & 08 & - \\
29 & 14 & 10 & - \\
30 & $\mathbf{0 9}$ & 06 & Emphysema \\
\hline
\end{tabular}

Maximum and minimum blood loss are made bold

and life threatening. Complications of PcT, like paratracheal insertion of tracheostomy tube were reported $0.8 \%$ incidence by Powell et al. [14], $0.6 \%$ by Moe et al. [15] and $0.5 \%$ by Lim et al. 16 . This complication may be due to calcification of tracheal rings especially in elderly patients.

When such life threatening complications occur, their management may needed to convert PcT to Conventional Surgical Tracheostomy [17].

\section{Conclusion}

In our study differences in Conventional Tracheostmy and Percutaneous Tracheostomy are statistically NOT significant. The best strategy would be, Percutaneous Tracheostomy is to be planned by properly trained hands, in patients who are already having endotracheal intubation, without any soft tissue neck and cervical injury. One 
Table 3 Operative time (in min) and blood loss (in ml) in group 1 and group 2

\begin{tabular}{lll}
\hline & Gp 1- Conventional T & Gp-2- Percutaneous T \\
\hline Operative Time (in min) & & 15 \\
Samples & 15 & $09-54$ \\
Range & $04-41$ & 23.933333 \\
Mean & 16.400000 & 10.361099 \\
SD & 10.662350 & 10.512804 \\
Overall SD & -1.962453 & 15 \\
t value is & 28 & $04-16$ \\
Degree of freedom is & 0.059717 & 8.066667 \\
$p$ value is & & 3.594970 \\
Blood loss (in ml) & 15 & $04-12$ \\
Samples & 6.800000 & \\
Range & 2.274078 & \\
Mean & 3.007926 & -1.153256 \\
SD & 28 & 0.258555 \\
Overall SD & & \\
t value is & & \\
Degree of freedom is & & \\
$p$ value is & &
\end{tabular}

Result is NOT significant for $5 \%$ level

should always remember that which ever technique is opted, it should be beneficial to the patient. Also cost factor of PcT has to be considered. It is suggested that while considering PcT, possibility of need of conversion of PcT into Conventional Surgical Tracheostomy should always be kept in mind and an ENT surgeon, who is confident to manage complications of PcT by virtue of their training in Conventional Surgical Tracheostomy should be present during the procedure.

It is of utmost importance in a critically ill patient that, whatever is the indication, which ever technique/approach for tracheostomy is adopted, whoever is doing the tracheotomy, it should be after careful assessment of patient, considering all the factors discussed and it is to be done with high success rate with No and/or minimum frequency and severity of complications and should be beneficial to the patient, his family and thus to the society at large.

\section{What It Adds to Existing Information in the Field?}

Person with refined skill in the technique, is of utmost importance in deciding the choice of a technique.

Acknowledgements Our sincere acknowledgements to the members of institutional ethics committee, the Management, Dept. of anaesthesiology. Dr. Arvind Sir (Professor. PSM), Everyone in the Intensive Care Unit and our patients.

\section{References}

1. Shelden C, Pudenz RH (1957) Percutaneous tracheotomy. JAMA 165:2068-2070

2. Ciaglia P, Graniero KD (1992) Percutaneous dilatational tracheostomy. Results and long-term follow-up. Chest 101:464-467

3. Ciaglia P, Firsching R, Syniec C (1985) Elective percutaneous dilatational tracheostomy. A new simple bedside procedure; preliminary report. Chest 87:715-719

4. Schachner A, Ovil Y, Sidi J et al (1989) Percutaneous tracheostomy-a new method. Crit Care Med 17:1052-1056

5. Griggs WM, Myburgh JA, Worthley LIG (1991) A prospective comparison of a percutaneous tracheostomy technique with standard surgical tracheostomy. Intensive Care Med 17:261-263

6. Head JM (1961) Tracheostomy in the management of respiratory problems. N Engl J Med 264:587-591

7. McLelland RMA (1965) Complications of tracheostomy. Br Med J 2:567-569

8. Mulder DS, Rubush JL (1969) Complications of tracheostomy: relationship to long-term ventilatory assistance. J Trauma 9:389-402

9. Chew JY, Cantrell RW (1972) Tracheostomy. Complications and their management. Arch Otolaryngol 96:538-545

10. Skaggs JA, Cogbill CL (1969) Tracheostomy: management, mortality, complications. Am Surg 35:393-396

11. Marelli D, Paul A, Manolidis S et al (1990) Endoscopic guided percutaneous tracheostomy: early results of a consecutive trial. J Trauma 30:433-435

12. Yarington CT, Frazer JP (1965) Complications of tracheotomy. Arch Surg 91:652-655

13. Donaldson DR, Emami AJ, Wax MK (2000) Endoscopically monitored percutaneous dilational tracheostomy in a residency program. Laryngoscope 110:1142-1146 
14. Powell DM, Price PD, Forrest LA (1998) Review of percutaneous tracheostomy. Laryngoscope 108:170-177

15. Moe KS, Schmid S, Stoeckli SJ, Weymuller EA (1999) Percutaneous tracheostomy: a comprehensive evaluation. Ann Otol Rhinol Laryngol 108:384-391
16. Lim JW, Friedman M, Tanyeri H, Lazar A, Caldarelli DD (2000) Experience with percutaneous dilatational tracheostomy. Ann Otol Rhinol Laryngol 109:791-796

17. Meade JW (1961) Tracheotomy-its complications and their management. A study of 212 cases. N Engl J Med 265:519-523 